\title{
The Development of Tik Tok's Global Market
}

\author{
Yuchen Ren ${ }^{1, a,{ }^{*}}$ Wanxin $\mathrm{Qi}^{1, \mathrm{~b}}$ Mingyang Fan ${ }^{1, \mathrm{c}}$ \\ ${ }^{a}$ College of Letters and Science, University of California, Santa Barbara, Goleta, California, 93117, USA \\ ${ }^{b}$ Nanwai King's College School, Wuxi, Jiangsu Province, China \\ ${ }^{c}$ Wenshan Middle School, Weifang, Shandong Procince, China \\ *Email: steven.ren1229@gmail.com
}

\begin{abstract}
Tik Tok creates a worldwide mania, becomes one of the most popular and successful social media platforms. The success of Tik Tok cannot take place without its strong algorithm. In combination with knowledge of Business Analytics and Economics, this essay measures how Tik Tok develops its global market with statistical data. This study collected and analyzed people's experience of using Tik Tok through questionnaire surveys. . In the end, as a result, we conclude that Tik Tok's algorithm integrates CVM to maintain a wide customer base, then expands globally, and makes impacts on the economy.
\end{abstract}

Keywords: Tik Tok, CVM, Market, Economy, Algorithm

\section{INTRODUCTION}

The ByteDance launched Tik Tok, which is a music video platform for young people, it rapidly expanded and became one of the most popular applications in the world. For its users, it provides a platform for young people to express themselves, fill in fragments of leisure time, and obtain information. For business, Tik Tok creates a new business model based on its platform. It links social media, online retail, and video sharing platforms to create unique value.

Tik Tok uses artificial intelligence algorithms to make targeted content recommendations to customers based on the habits and tastes of people in different regions. At the same time, users are classified according to different markets to obtain higher customer stickiness. In terms of expanding business value, Tik Tok cooperates with the brand and provides it with an online retail platform and users.

The first part of this research uses questionnaire surveys to analyze the impact on the customers and market trends by using of Customer Value Measures (CVM) and algorithm. This paper also presents the market strategy of Tik Tok in expanding its global market. Due to the algorithm it used, the potential of Tik Toks commercial values still be competitive. However, based on the current competition in the short video market, Tik Tok can consider establishing cooperation with other industries when expanding its global market, such as the financial industry. Thereby, it can broaden the scope of services of its platform, and further Increase its commercial value.

\section{APPLICATION OF CVM IN TIK TOK}

Tik Tok's appearance provided a brand-new platform of social media, which attracts millions of users all over the world. Viewers have various preferences (Figure 1), and the capability of Tik Tok's comprehension for each viewers' preferences is its key to its worldwide success. In combination with Data Science algorithms, Tik Tok grasps each customers' values to its business precisely, makes accurate predictions, and creates a large customer base for generating profits.

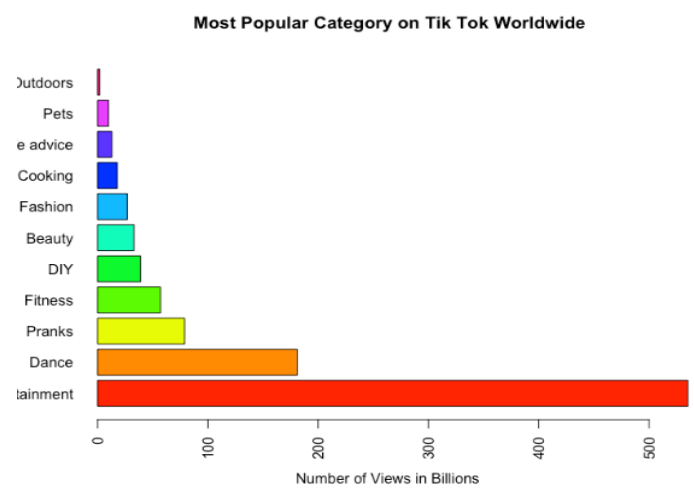

Figure 1 Most Popular Category on Tik Tok Worldwide [2] 


\subsection{What is CVM?}

CVM stands for "Customer Value Measures", and CVM can be interpreted from two perspectives: the business and the customer. From the business's side, CVM demonstrates the value that customers individually bring to the business, based on the profits that each individual customer contributes to the business. On the other hand, for customers, CVM represents how much value of the product or the service provided by the business is to the customers. Specially, will the values of the product or the service can establish an emotional bond between the customers and the business [1].

\subsection{CVM and Algorithm in Tik Tok}

Tik Tok attracts influencers to post videos on its platforms with diverse contents, which fulfills the social media platform with multiple categories. The top 3 categories worldwide are Entertainment, Dance, and Pranks, based on number of views [2]. One of the ways for Tik Tok to generate profits is to promote advertisements. Since customers prefer to view contents regarding Entertainment, Dance, and Pranks, most of the advertisements will be played in between videos under those categories. Moreover, the content played for each user on Tik Tok shifts based on one's interests.

Customer bonding is to build up customers' reliance on the products or the service that the customer values [1], which is significant for Tik Tok's CVM. Tik Tok utilizes Artificial Intelligence as part of its algorithm for accomplishing this goal. What is unique about Tik Tok's algorithms is that it learns users' individual preference within time. As in time, the algorithms will be able to make precise predictions on the categories of the video for each individual; thus, the customer develops reliance on Tik Tok which completes the construction of the customer bonding.

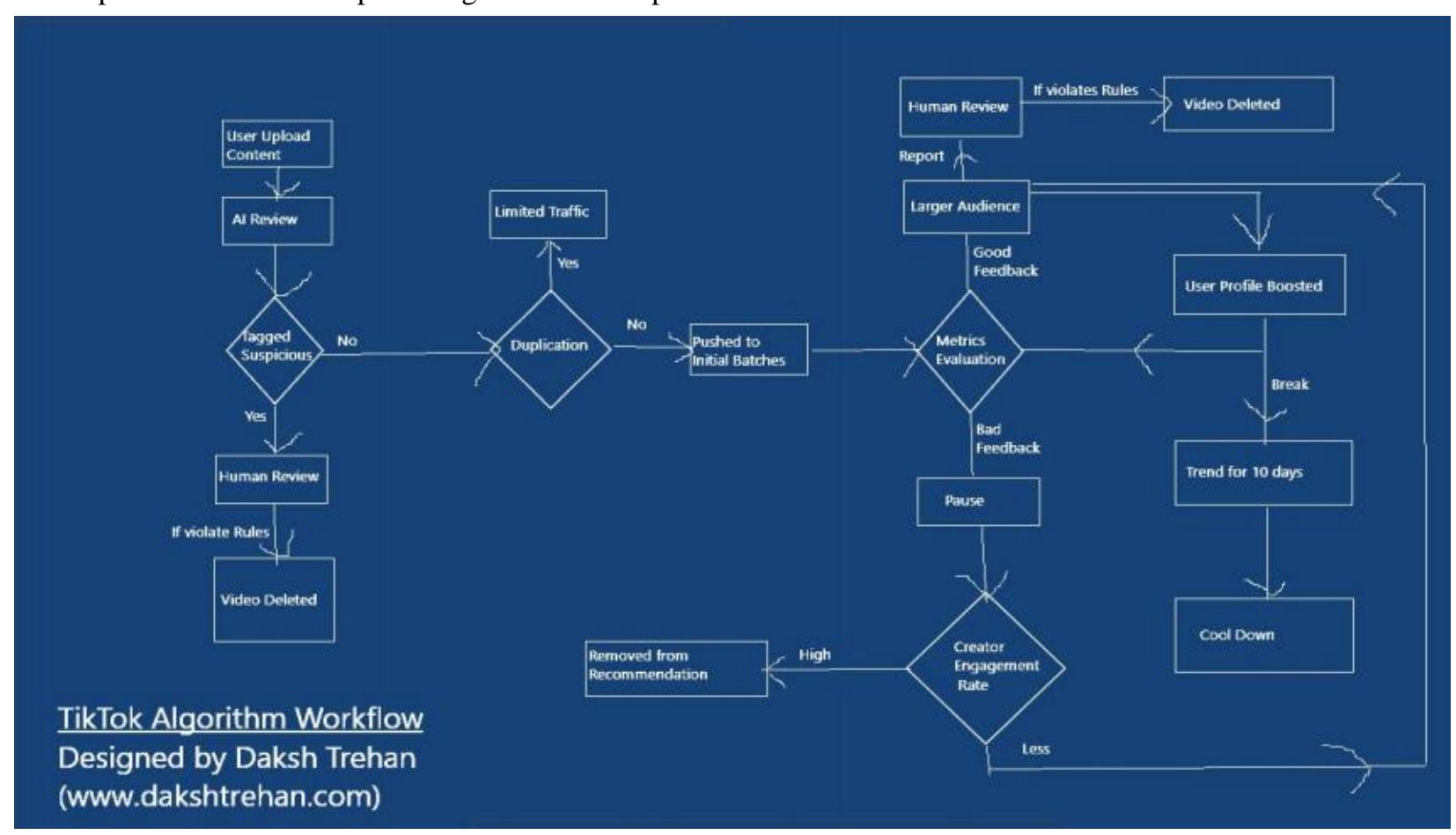

Figure 2 Tik Tok Algorithm Workflow

Experts suggest a possible theory behind the algorithms of Tik Tok on how it works for valuing each customer (Figure 2). It is highly likely that Tik Tok's algorithm has a metric rating system for every action that one could possibly take, and the algorithm is smart enough to be able to detect excessive actions happening. For instance, if the customer re-watched a video, the customer received 10 pints, 8 points for comments or sharing the video with other people [3]. Eventually, after cycles of cycles of the evaluation system, this completes Tik Tok's Recommender System, which mainly recommends contents based on one's preference.
Nowadays, many influencers sell products on Tik Tok, and Tik Tok will take a percentage of commission fee from the influencers. On the basis of the Recommender System established by the algorithms for each individual customer, Tik Tok, now, knows what kind of the products each customer values the most, and which influencers bring most profits to Tik Tok through selling products. Therefore, the platform will recommend videos containing products that may satisfy customers' potential needs that the customer will most likely make a purchase. Besides, the platform will also recommend videos or live streams of influencers with higher revenue. 


\subsection{Research on the Influence of Tik Tok's Algorithms}

The goal for of this research is to find out the effect of Tik Tok's algorithms on users' experience. The research method was using a questionnaire created using Google Form. The questionnaire was posted on WeChat and Instagram. In the end, 51 responses were received.

Questions on the Google form consisted of two parts: basic information and questions on Tik Tok. Basic information contains Country/Region, Sex, Age, and Occupation. Questions regarding their experience with Tik Tok:

1. How often do you use Tik Tok?

2. Have you shopped on Tik Tok before? Tok?

3. What is your favorite content category on Tik

4. Do you think Tik Tok mainly recommends videos of your interest?

\subsubsection{Results}

The 51 responses consist of people from China (21), U.S(16), UK(3), Philippines(9), and France(2). 54.9\% are females, and $45.1 \%$ are males. The respondents' age ranges from 16 to 42 . $56.8 \%$ of the participants are students (high school and college students), the rest of the $43.2 \%$ participants are working, but choose not to disclose their occupation.

Nearly $96.07 \%$ of the participants use Tik Tok daily, only 2 people responded do not use Tik Tok daily. This result reflects the high frequency of the usage of Tik Tok in people's daily lives at the moment, as matter of fact, overall Tik Tok has 689 million users monthly worldwide [4].

All 21 participants from China shopped on Tik Tok at least once, which consists of 41 percent of the participants. One person from the U.S and 2 people from the U.K also shopped on Tik Tok before for at least once. Participants responded that they have brought Cosmetics, Pet Supplies, Food, Clothing, and Housing Supplies.

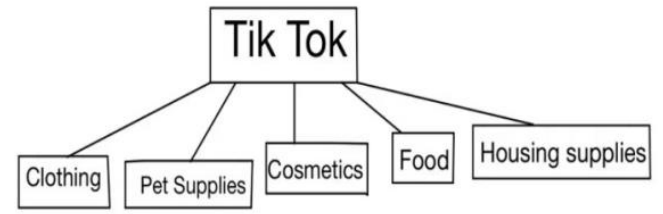

Figure 3 Popular Items Participants Brought on Tik Tok

An interesting fact based on the participants who had purchased products on Tik Tok before, those participants also liked to watch videos on Tik Tok for those categories. The categories include Fashion, Pet,
Beauty, Cooking, and DIY(Figure 3). Influencers who post videos in those categories also sell products on Tik Tok, and participants normally watch those influencers at a high frequency. Participants also stated that if they have purchased a product on a specific influencer's account, then, he or she receives that influencer's video a lot and other influencers' videos of the same category. $88.23 \%$ of the participants do agree that Tik Tok mainly recommends videos of his or her interest. Particularly, they felt that the longer they use the app, the more videos of his or her interests were recommended by Tik Tok.

\subsubsection{Summary}

Based on the results of the research from 51 responses, it is clear that Tik Tok's algorithm contains a Recommender System for each individual user. The algorithm is capable of predicting videos that may intrigue the user at high accuracy. Eventually, Tik Tok can identify each customer's value on different videos or different products; also, Tik Tok can perceive valuable customers who make the most purchases, then more videos with products to sell will be sent to the viewer. However, the 51 responses are still limited to the study, but the findings are compelling. Data with larger participants will be more beneficial for studying the effect of Tik Tok's Algorithms on its users.

\section{THE EXISTING TIK TOK'S BUSINESS STRATEGY}

\subsection{Overseas merger and cooperation}

In order to obtain a wide market in the United States, Toutiao purchased the original Apps, "Flipagram" and "Musical. ly". Tik Tok use the wide range of customers of these two Apps to increase their own users. This action helps eradicate a strong competitor of Tik Tok, and creates a lot of opportunities to cooperate with World Media Group [6].

\subsection{Cater to the user}

The reason why the App "Musical. ly" is popular is that it satisfies the local users' needs in America. It clearly knows its users and makes the App's operating interface easy to use. "Musical. ly" gives the user the freedom to create rich content. Also, "Musical. ly" organizes parties for the users regularly which provides a platform for the users to communicate about their experience of creating short videos. Tik Tok learns and study the operation mode of "Musical. ly", on this basis of innovation and transformation, and referenced to other countries.[5]. 


\subsection{Invite celebrities to settle in}

Countries like Japan has a conservative idea. It is hard to open up a sustainable market in these countries. Tik Tok uses a different strategy. The Tik Tok domestic team finds celebrities matching the team design theme, and ask them to create freely under different themes. After having these celebrities for creating, the team also takes the mode of signing artist to attract customers [5].

\subsection{Target on the customers}

The key for media to obtain a large amount of audience is to win the customers' love. The Tik Tok team segment the customers. Clealy know the total global population, ranking, age, and distribution of gender structure. Take the advantage of different countries' population to maximize its users.[7]

\section{THE IMPACT OF TIK TOK'S OVERSEAS MARKET}

After integrating its commercial measures, Tik Tok began to enter overseas markets. On July 16, 2018, the global monthly active user data was officially released for the first time, with more than 500 million monthly active users worldwide. According to data from American research firm Senser Tower, in the first quarter of 2018, Tik Tok was the world's most downloaded iPhone application, with 45.8 million downloads, surpassing Facebook, You Tube and Instagram. In addition, Tik Tok has frequently achieved higher rankings in APP download rankings in Japan, Thailand, and Indonesia. Tik Tok covers more than 150 countries and 75 languages, ranking first in the app store in more than 40 countries and regions [9].

\subsection{Contents and preferences of overseas users}

Tik Tok has a powerful algorithmic recommendation mechanism and artificial intelligence technology. Through the collection of local cultures, styles and user preferences in different regions, it analyzes user portraits and accurately pushes video content that meets user preferences. Through the algorithmic recommendation system, according to the user's use behavior to meet the user's personalized needs to enhance user stickiness. Not only that, the platform will also launch different fun stickers according to the major festivals of each country to strengthen the past cultural exchanges between countries and promote the diversity of the world [9].

\subsection{New forms of entertainment dissemination}

Tik Tok is spread in the form of short videos. It breaks the traditional form of graphics transmission. A far cry from Youtube is that Tik Tok has a powerful music library, and the video length is short and precise. In addition, Tik Tok has a simple editing method that attracts many people who know little about editing and creation.

Tik Tok is a platform to spread national culture. Short videos are a simpler and more attractive way. It breaks the language barrier. With the arrival of celebrities, Tik Tok has become a phenomenon-level local communication product.

\section{TIK TOK'S COOPERATION WITH THE OTHER INDUSTRY - FINANCIAL INSTITUTION}

Short video has exploded since 2016 and is now an indispensable medium for the consumption of entertainment content and information. According to Figure 4, the short video market scale was valued at 19 billion in 2016 and reached 467.1 billion in 2018, and is expected to reach 2110.3 billion in 2021. With the gradual popularization of the mobile Internet, the short video industry is also in line with the trend of the times more vigorous upward. Due to the increase in the number of Tik Tok users, Tik Tok needs to find new business cooperation opportunities except for the general business cooperation. Financial institutions are seeking co-operation with short video platforms.

According to the ]the Ocean Engine, the short video industry in 2021 is still in a high-speed development stage, and even some brand-new platforms and marketing plays continue to enter the game. A short video is a necessary platform for financial institutions as it needs high exposure and coverage. However, it requires understanding the trends and opportunities early to set up a marketing strategy for this track.[10]

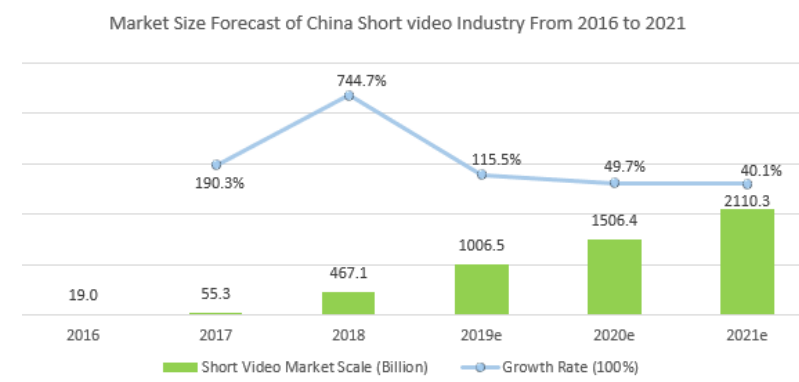

Figure 4 Market Size and Prediction of Short-Video Industry in China: 2016-2021 [10]

According to Tik Tok's Financial Content Eco Report (Figure 5), the platform data presented for the period from November 2019 to November 2020, overall, has three sets of data to focus on[10]. The first set of data are in November 2020, the number of people interested in Tik Tok's Finance reached 105 million. The second is that proportion of financial users of the Tik Tok's platform, $53.3 \%$ of users with financial 
behavior, Unfinished users accounted for $46.7 \%$ The third set of data are the penetration rate of financial products financial management users of Tik Tok's platform, Insurance $38.4 \%$, IMF $32.6 \%$, Bank Wealth Management $29.1 \%$. According to a report, in November 2020, the number of people with financial interest reached 1.05 billion, the distribution of financial interest users is relatively balanced, users of all ages and cities are growing rapidly, high-line cities, new generations and older generations have stronger preferences. Therefore, whether from the introduction of property products or investment philosophy is a good medium.[10]

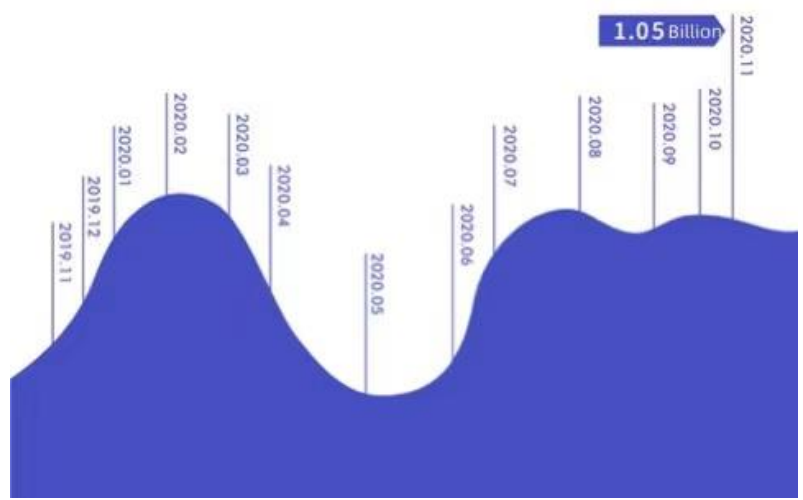

Figure 5 The number of people with financial interest

[10]

From the point of view of gender, Tik Tok's financial interest users sex ratio of 57:43, male users slightly ahead of the growth rate, women's growth space is worth looking forward to (Figure 6).

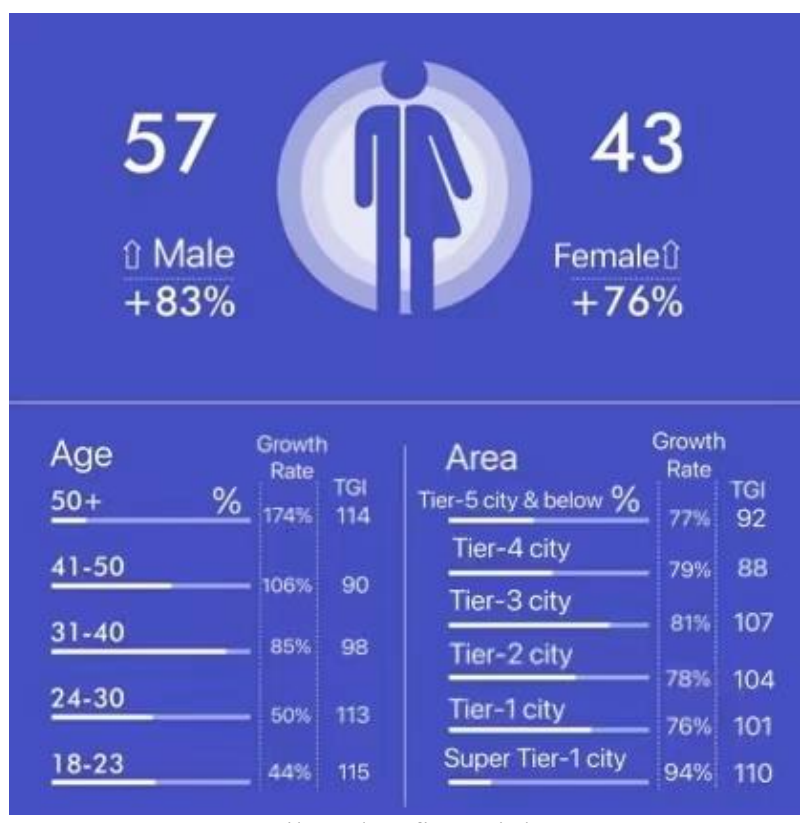

Figure 6 Tik Tok's financial users [10]

From the Figure 7, we can find that whether from the market size or people's needs, to shake the tone as a medium to spread financial products is a very good innovation. Tik Tok's platform financial users accounted for: financial behavior users accounted for $53.3 \%$, unfinished financial users accounted for $46.7 \%$, from the proportion can be seen that the growth of Tik Tok's platform financial users have a huge space, Tik Tok's platform financial products financial user penetration rate: insurance $38.4 \%$, money fund $32.6 \%$, bank wealth management products $29.1 \%$, the total penetration rate of these three blocks of $100.1 \%$, the most popular Tik Tok's user.[10]

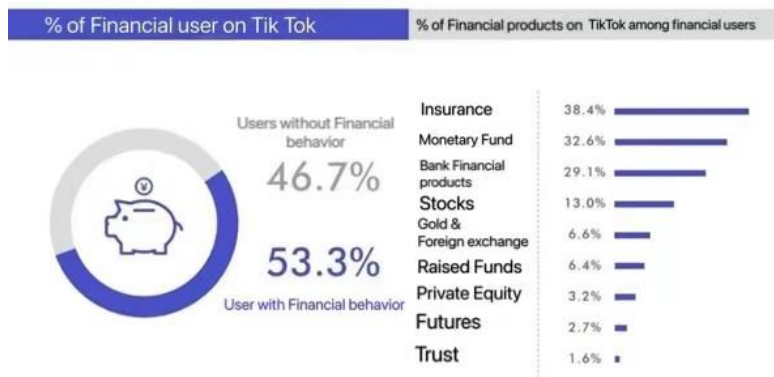

Figure 7 the percentage of Financial user and products on Tik Tok

\section{CONCLUSION}

Through the research we did in this paper, it is obvious that the Recommend System for each individual user is significant for Tik Tok's algorithm. Tik Tok, as a new type of App, uses the technology of AI as part of its algorithm for its real-time user monitoring. Accurately detect the customers' preferences and recommend the videos to the customers according to the data obtained from the algorithm. It attracts many users with the help of a special algorithm.

In order to expand the global market, Tik Tok follows the rule that adjusting measures to the local condition. Tik Tok teams make different strategies targeted at different countries. With the cooperation and merger of the brand. It obtained a wide market and users based on the original platforms provided by the brand. With the help of algorithm, Tik Tok quickly occupies an important position in the global market. Although they still have lots of problems to face, the achievement they have had is spectacular.

Under the strong platform, Tik Tok provided, it has made a great contribution to the economy. Many users use the advantage of short videos to promote their products. This kind of promoting and shopping has become the most popular form among users, which promotes economic growth. As the number of downloads is still rapidly increasing, the amount of people who buy financial products and goods has a huge space to grow. Tik Tok could consider cooperating with the financial organization while expanding the market and achieve a win-win situation.

Although there is a strong sound that Tik Tok should be out of the overseas market in foreign country, Tik 
Tok still achieved a great achievement. This paper analyzes the marketing and business strategy of Tik Tok, hoping to help Tik Tok achieve greater success in the future.

\section{REFERENCES}

[1] Jr.Butz, E. Howard and Leonard D. Goodstein. "Measuring customer value: Gaining the strategic advantage." Organizational Dynamics, Volume 24, Issue 3, 1996, Page 63-77, https://www.sciencedirect.com/science/article/pii/S 0090261696900066 . Accessed 22 August 2021.

[2] Statistia Research Department. "Most popular content categories on TikTok worldwide as of July 2020 , by number of hashtag reviews." Statistia, July. 2020 , https://www.statista.com/statistics/1130988. Accessed 21 August 2021.

[3] Trehan, Daksh. "The incapable Al Algorithm: TikTok." TowardsDataScience, 12 Jun. 2020, https://towardsdatascience.com/the-inescapable-aialgorithm-tiktok-ad4c6fd981b8.Accessed 20 August 2021.

[4] Iqbal, Mansoor. "TikTok Revenue and Usage Statistics (2021).” Business of apps, 4 Aug. 2021, https://www.businessofapps.com/data/tik-tokstatistics/. Accessed 21 August 20

[5] Li Xin, And Li Deseng." Overseas communication strategy of Short video "Tik Tok" : A case study of the United States, Japan and Southeast Asia." Journal of Beijing Institute of Graphic Technology 29.01(2021):5-7. doi:10.19461/j.cnki.10048626.2021 .01 .002

https://kns.cnki.net/kcms/detail/detail.aspx?dbcode $=\mathrm{CJFD} \&$ dbname $=$ CJFDLAST2021\&filename $=\mathrm{BY}$ XB202101002\&v=Ohhi2tOuoIAKsn1tlm6UaMnw
BNOMO\%25mmd2BYitxJ5d5\%25mmd2BqiqTxI Dvg9mQsHjxx\%25mmd2BWImWuuJ

[6] Huang Jie. "Research on the path and Strategy of Chinese media "going out"--Taking TikTok, the international version, as an example." Voice \& Screen World. 06(2021):64-65+92. doi:CNKI:SUN:SPSJ.0.2021-06-028.

https://kns.cnki.net/kcms/detail/detail.aspx?dbcode $=$ CJFD $\&$ dbname $=$ CJFDLAST2021 $\&$ filename $=$ SP SJ202106028\&v=SjyqLs640jkNkVQprMRP9fooR $\% 25 \mathrm{mmd}$ 2BpdDfieill0ZEL4O1919I45KKNMsNtF9 w02HiC5

[7] Zhang Chunlin.'On the relationship between media audience strategy.' Contemporary Communication.05 (2006):5052.doi:CNKI:SUN:DACB.

https://kns.cnki.net/kcms/detail/detail.aspx?filenam $\mathrm{e}=\mathrm{DACB} 200605019 \& \mathrm{dbcode}=\mathrm{CJFQ} \& \mathrm{dbname}=\mathrm{cjf}$ d2006\&v=iGZag0_8-tD4jXVpw 4PeAAOVdp3rfaXNU4Nxy99XH5F9CT2KyxEU mutpas0M7VR

[8] Cheng Y. "Analysis on the difficulties and strategies of Douyin entering overseas." Journal of News Research 11.12(2020):204-205. doi:CNKI:SUN: xwDK.0.2020-12-124.

https://kns.cnki.net/kcms/detail/detail.aspx?dbcode $=$ CJFD \&dbname $=$ CJFDLAST2020 \& filename $=X$ WDK202012124\&v=SSiLaRBB7h7\%25mmd2Fz N9KgCTq3yUSKzY50iqIz0x2KCdNY2OYnDrHa OZZnOi\%25mmd2F7yUC8HQ3

[9] Zhou Xue." The overseas dissemination of the short video and the study of the self-plasticization of the national image." Audiovisual .09 (2019): 151-152. doi:10.19395/j.cnki.1674-246x.2019.09.085.

[10]The Ocean Engine, '2020 Tik Tok's Financial Content Eco Report.' www.oceanengine.com/insight/541 